\title{
Bandverletzungen des Ellenbogengelenks
}

\author{
Heinz Kusche, Peter Gutsfeld, Volker Bühren
}

\section{Zusammenfassung}

Bandverletzungen des Ellenbogengelenks sind meist Folge eines Luxationsereignisses. Einfache Luxationen ohne wesentliche Begleitverletzungen haben immer eine Ruptur des radialen Bandapparats zur Folge und heilen konservativ, meist folgenlos, nach Reposition aus. Mit zunehmender Schwere des Traumas und Komplexität der Verletzung mit weiteren, oft knöchernen Begleitpathologien, steigt die Rate an unbefriedigenden Ausheilungsergebnissen. Aus diesem Grund ist eine subtile Diagnostik und Klassifizierung der Verletzung mit stadiengerechter Therapie von entscheidender Bedeutung. Um die gefürchteten Arthrofibrosen des Gelenks zu vermeiden, ist das Ziel der Behandlung, ein stabil geführtes, kongruentes Gelenk zu schaffen, um eine frühzeitige Bewegungstherapie einleiten zu können.

\section{Ligament Lesions of the Elbow Joint}

Ligament lesions of the elbow joint are mostly caused by dislocations. Simple dislocations without major collateral damage always lead to a rupture of the radial ligamental structures. After closed reduction and non-operative treatment most injuries heal without any consequences. With increasing severity of the trauma and the associated complexity of the injury, which frequently also involves bone lesions, the rate of unsatisfactory outcomes increases. Therefore a correct diagnosis and classification of the severity of the injury is essential. A correct, classification-based treatment strategy is essential. The goal of the treatment is to create a stable and correctly articulating joint. This is the precondition for early functional rehabilitation to prevent arthrofibrosis of the elbow joint.

\section{Einleitung}

Ellenbogenluxationen stellen die zweithäufigste Luxationsform, beim Heranwachsenden die häufigste Luxation des Bewegungsapparats dar. Sie treten mit einer Inzidenz von 6-13/100000 Einwohner auf, werden am häufigsten als Folge von Sportunfällen diagnostiziert. Chronische Instabilitäten können aus einer nicht suffizienten Primärtherapie resultieren und erfordern aufwändige und technisch schwierige sekundäre Rekonstruktionen. Die Kenntnis der anatomischen Gegebenheiten, der möglichen Pathologien und des Spektrums der sinnvollen konservativen und operativen

OP-JOURNAL 2012; 28: 48-56

(c) Georg Thieme Verlag KG Stuttgart · New York DOI http://dx.doi.org/10.1055/s-0031-1298490
Das Humeroulnargelenk stellt den wesentlichen Stabilisator gegen Varusinstabilität in Streck- und Beugestellung dar, unterstützt wird dieses ligamentär durch den LCL-Komplex. Die Stabilisierung gegen Valgusinstabilität erfolgt in gleichbedeutenden Anteilen durch den medialen Bandkomplex, die ventrale Kapsel und die knöchernen Strukturen. Hierbei liegt der Anteil des Radiusköpfchens an der Stabilisierung gegen Valgusinstabilität bei $30 \%$, er erhöht sich durch Rupturen des MUCL auf ca. 75\%. Einen wesentlichen Stabilisator gegen nach dorsal gerichtete Kräfte stellt der Processus coronoideus dar. Als dynamische Stabilisatoren wirken die gelenkübergreifenden Muskeln M. biceps, M. triceps, M. brachialis und der M. anconaeus.

\section{Diagnostik}

\section{Klinische Untersuchung}

Die klinische Untersuchung gibt Hinweise über das Verletzungsbild, insbesondere Luxationsereignisse können durch eine auffällige Konturveränderung und Funktionseinschränkung des Gelenks festgestellt werden. Die Untersuchung sollte eine Befunderhebung der angrenzenden Gelenke, insbesondere des Handgelenks, einschließen, um bspw. eine Essex-Lopresti-Läsion mitzuerfassen. Palpationsschmerz über dem Kollateralbandverlauf und den exponierten knöchernen Strukturen kann Hinweise auf Läsionen im entsprechenden Bereich liefern. Eine wesentliche Bedeutung kommt der Evaluierung evtl. Gefäß-Nerven-Schädigungen zu. Verletzungen der A. brachialis werden mit einer Häufigkeit von $0,47 \%$, entsprechend bei etwa jeder 200. Luxation des Ellenbogens, beschrieben. Wegen guter Kollateralkreisläufe kann eine ausreichende Perfusion des Unterarms, trotz Verletzung der A. brachialis, möglich sein. Bei fraglichen Befunden mit Verdacht auf Gefäßschädigung sollte unverzüglich eine entsprechende Gefäßdarstellung erfolgen. 


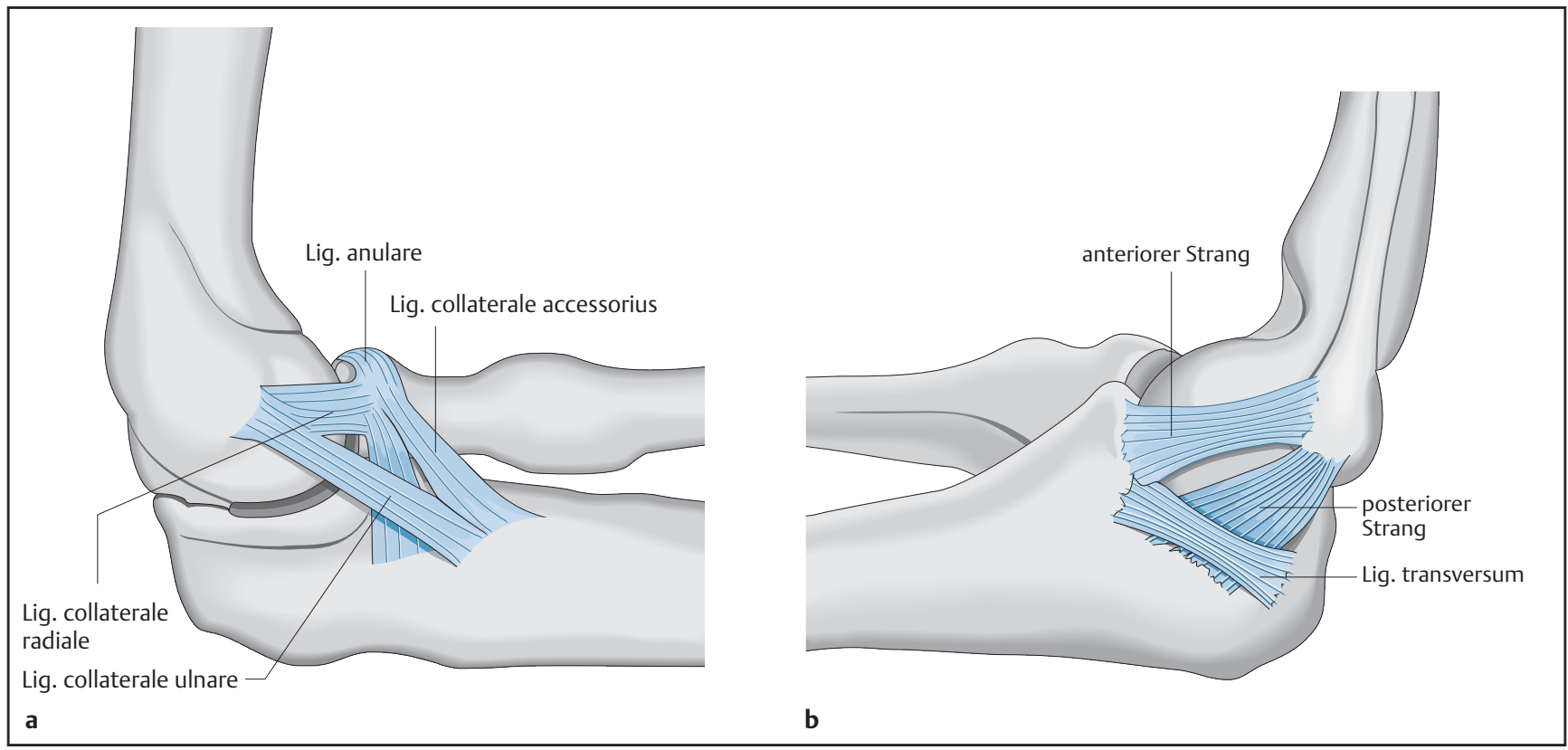

Abb. 1 a und b a Radialer Bandapparat (aus: Miller, Howard, Plancher. Operationsatlas Sportorthopädie - Sporttraumatologie. 2003. S. 413. Mit freundlicher Genehmigung des Elsevier Verlags, Oxford). b Ulnarer Bandapparat (aus: Miller, Howard, Plancher. Operationsatlas Sportorthopädie Sporttraumatologie. 2003. S. 420. Mit freundlicher Genehmigung des Elsevier Verlags, Oxford).

Motorische oder sensible Einschränkungen sollten vor Durchführung diagnostischer und therapeutischer Maßnahmen subtil erfasst und dokumentiert werden. So wurden bei kindlichen Ellenbogenluxationen Irritationen des N. ulnaris in bis zu 21\% der Fälle beschrieben, Beeinträchtigungen des Medianusnervs in bis zu 12,5\%. Beschreibungen über Läsionen des N. radialis beschränken sich auf Einzelfälle. Nach erfolgter Reposition sind die neurologischen Irritationen zumeist nach wenigen Wochen komplett regredient.

\section{Radiologische Diagnostik}

Als Standarddiagnostik gilt die Röntgendarstellung des Ellenbogens in 2 Ebenen. Hiervon abhängig kann das weitere Vorgehen geplant werden. Bei Schmerzen im Handgelenksbereich wird die ergänzende Röntgendiagnostik des Handgelenks zum Ausschluss der oben genannten Essex-Lopresti-Läsion, mit Ruptur der Membrana interossea und Instabilität im distalen Radioulnargelenk, empfohlen.

Bei knöchernen Verletzungen wie einer Absprengung des Processus coronoideus, knöchernen Bandausrissen oder Radiusköpfchenfrakturen wird eine Darstellung des Gelenks mittels CT empfohlen.

Eine 3-D-Darstellung kann hierbei zur präoperativen Planung hilfreich sein. Ge- gebenenfalls ist eine Gefäßdarstellung mittels CT-Angiografie im Rahmen der Untersuchung sinnvoll. Die Indikation zur Gefäßdiagnostik sollte großzügig gestellt werden, eine einfache und kostengünstige Methode stellt hierzu die Doppler-Sonografie dar. Die Kernspintomografie ist selten bei akuten Verletzungen erforderlich, sondern hat ihre Berechtigung eher bei der Beurteilung chronischer, vor allem medialer Bandinstabilitäten, gegebenenfalls auch bei Patienten im Wachstumsalter zur Darstellung okkulter Frakturgeschehen.

Nach erfolgter Reposition wird die funktionelle Untersuchung unter dem Bildverstärker empfohlen. Hierbei wird die Luxationstendenz bei Streckung und Beugung, insbesondere im Verlauf des "funktionellen Bogens" zwischen $30^{\circ}$ und $130^{\circ}$, beurteilt. Zudem sollte eine Überprüfung der Stabilität unter Valgusund Varusstress, jeweils in Streckung und $30^{\circ}$ Flexion, erfolgen.

Die Überprüfung der Stabilität unter Valgisierung sollte in Pronationsstellung durchgeführt werden, um nicht eine Pseudovalgusinstabilität falsch einzuschätzen. Die meist chronisch bestehende posterolaterale Rotationsinstabilität (PLRI) kann durch den lateralen PivotShift-Test identifiziert werden. Hierbei wird, beim auf dem Rücken liegenden Patienten, der Arm über den Kopf gehalten und in supinierter Stellung, bei axialer Kompression, unter zunehmendem Valgusstress gebeugt. Dabei zeigt sich bei positivem Testergebnis bei etwa $40^{\circ}$ Beugung ein Subluxationsphänomen, welches sich bei weiterer Beugung durch ein spürbares Schnappen reponiert (Abb. 2).

\section{Einteilung/Klassifikationen}

Die Luxation des Ellenbogengelenks läuft in 80-90\% der Fälle nach posterolateral ab. Der Verletzung liegt meist ein Sturz auf die extendierte Hand, bei gestrecktem Ellenbogen, zugrunde. Seltener treten rein posteriore, posteroulnare oder anteriore Luxationen auf. Bei der sehr seltenen divergierenden Luxation kommt es zur Zerreißung der Membrana interossea und zu einem Eintreten des distalen Humerus zwischen Radius und Ulna. Nach dem Modell von O'Driscoll nimmt die typische Ellenbogenluxation ihren Ausgang mit einer subtotalen bzw. totalen Ruptur des radialen Bandkomplexes. Mit zunehmender Krafteinwirkung kommt es zu einer weiter nach medial reichenden Läsion mit transossärem oder transkapsuloligamentären Verlauf. Im Stadium I kommt es zu einer (sub-) totalen Ruptur des lateralen ulnaren Kollateralbands und einer posterolateralen Subluxation des Ellenbogens. Im Stadium II sind zusätzlich die ventralen und dorsalen Kapselstrukturen verletzt und es kommt zu einem „Reiten“ des Processus coronoideus auf der Trochlea. Im Stadium III zeigt sich eine komplette Luxation des Gelenks. Hierbei besteht zusätzlich eine mediale Bandläsion. Im 
Stadium 3A sind die Zügel des anterioren medialen Kollateralbands jedoch noch intakt, im Stadium 3B ist der gesamte mediale Bandapparat rupturiert. Im Stadium 3C kommt es durch eine Läsion der langen Flexoren und Extensoren zu einer Separation des distalen Humerus und durch den Verlust der dynamischen Stabilisatoren zu einer hoch instabilen Situation des Gelenks. Die posteromediale Varusrotationsinstabilität beginnt mit einem Ausriss an der anteromedialen Fläche des Processus coronoideus und ist ebenso als hoch instabile Situation zu sehen (Abb. 3).

\section{Chronische Instabilitäten}

Mediale chronische Instabilitäten sind durch wiederholt einwirkende valgisierende Kräfte, häufig als Folge von Wurfsportarten, zu beobachten. Diese sind klinisch schwer zu evaluieren, da die Instabilität vom Patienten nicht immer dezidiert als solche empfunden und beschrieben wird. Die Untersuchung des medialen Bandapparats unter Valgisierung des Ellenbogens sollte in Pronationsstellung des Unterarms erfolgen. Laterale chronische Instabilitäten sind, bedingt durch die meist nach lateral weisende Luxationsrichtung, häufiger. Klinisch lassen sich chronische posterolaterale Rotationsinstabilitäten (PLRI) durch Subluxationsphänomene in strecknaher Position bei axialer Krafteinwirkung auf den supinierten Unterarm mit dem Pivot-Shift-Test nachweisen. Bei der PLRI Grad I führt der Test allenfalls zu einer minimalen Subluxation des Radiusköpfchens, Grad II beschreibt eine geringgradige Subluxation. Bei der drittgradigen PLRI zeigt sich eine deutliche Subluxation, Grad IV beschreibt eine Luxation des Radiusköpfchens. Ergänzend lassen sich die beschriebenen Instabilitätsformen unter dem Bildverstärker darstellen und dokumentieren.

\section{Knöcherne Begleitverletzungen}

In 20-50\% der Ellenbogenluxationen werden knöcherne Begleitverletzungen beschrieben. Diesen Läsionen kommt häufig eine entscheidende Rolle im Hinblick auf die Stabilität des Gelenks zu.

Frakturen des Processus coronoideus werden in bis zu 15\% der Luxationen beschrieben und nach Regan und Morrey in 3 Stadien eingeteilt. Diese Läsionen sind als Abscherverletzungen im Rahmen des Luxationsereignisses zu werten. Im Stadium I ist lediglich die Koronoidspitze

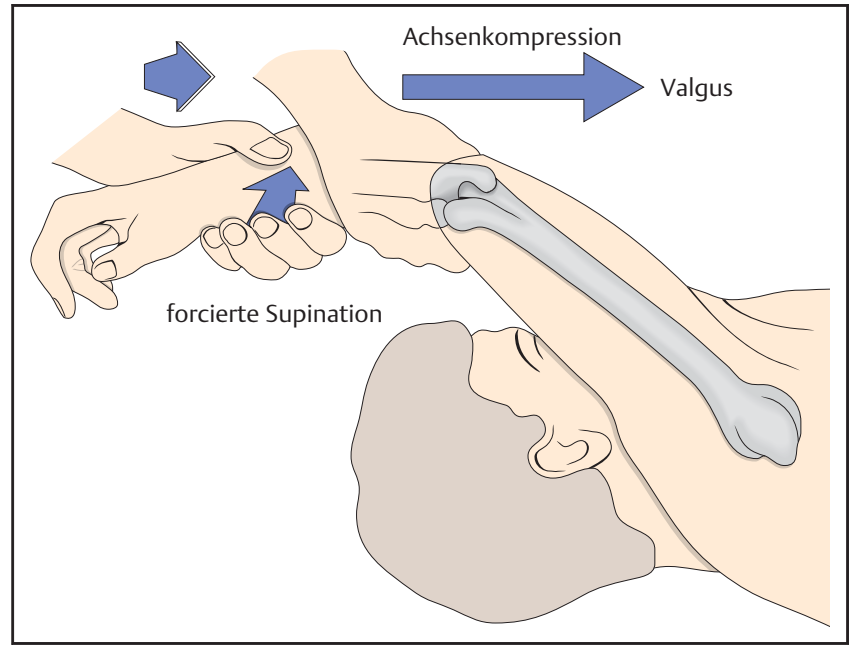

Abb. 2 Pivot-ShiftTest bei posterolateraler Rotationsinstabilität (PLRI) (aus: Miller, Howard, Plancher. Operationsatlas Sportorthopädie Sporttraumatologie. 2003. S. 413. Mit freundlicher Genehmigung des Elsevier Verlags, Oxford).

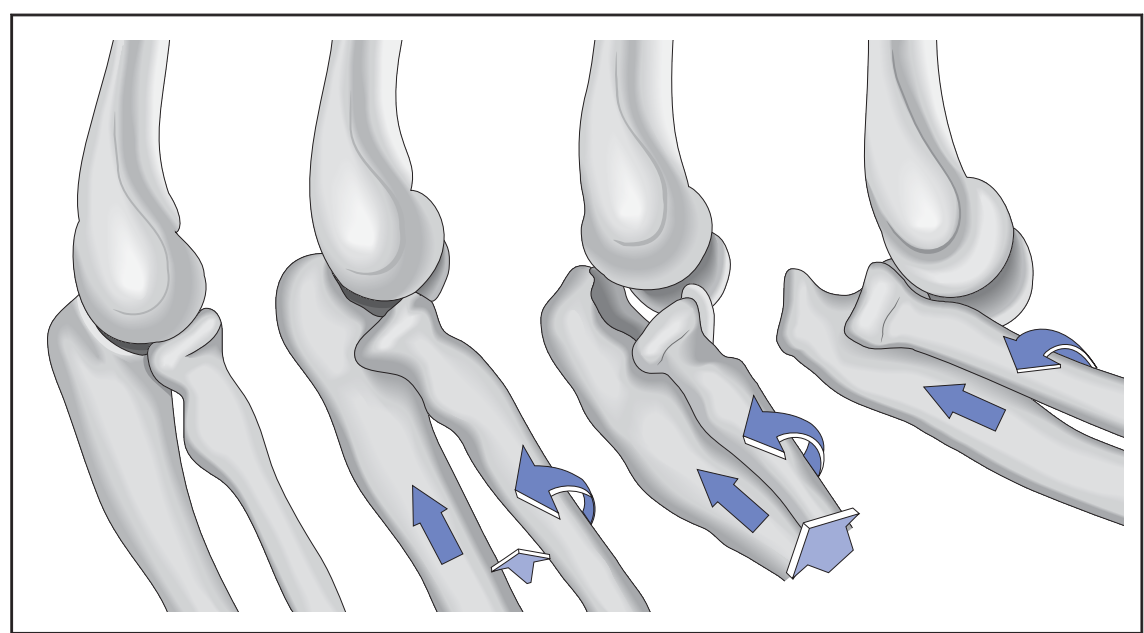

Abb. 3 Die 3 Stadien der Ellenbogengelenksluxation (aus: Miller, Howard, Plancher. Operationsatlas Sportorthopädie - Sporttraumatologie. 2003. S. 411. Mit freundlicher Genehmigung des Elsevier Verlags, Oxford).

frakturiert. Im Stadium II ist weniger als $50 \%$ der Höhe des Koronoids in seitlicher Projektion betroffen, im Stadium III mehr als 50\%. Die Bedeutung der Koronoidverletzung ist immer im Gesamtbild der Verletzung zu beurteilen. Als Zeichen einer erheblichen Instabilität gelten Abrisse eines anteromedialen Kantenfragments des Koronoids, da hier der Ansatz des kräftigen und für die Gelenkführung bedeutsamen anterioren Bündels des ulnaren Kollateralbands liegt. Bandinsuffizienzen in diesem Bereich haben posteromediale Instabilitäten zur Folge. Die Fragmente sind durch Überlagerungen im konventionellen Röntgen häufig nicht ausreichend abgebildet, sodass bei Auffälligkeiten in diesem Bereich eine CTUntersuchung zur exakten Befundevaluierung angestrebt werden sollte.

Radiusköpfchenfrakturen treten bei ca. 10\% der Luxationen am Ellenbogen auf und sind häufig Zeichen einer höhergradigen Instabilität des Gelenks. Sie können in Kombination mit ligamentären Verletzungen, insbesondere des medialen Bandapparats, einen erheblichen Instabilitätsfaktor darstellen. Sie werden nach Mason in Typ I-IV klassifiziert. Seltener zeigen sich Abrissfrakturen der Kondylen, Olekranonfrakturen oder Abscherverletzungen der Trochlea.

Als „Terrible Triad“ des Ellenbogens wird die Kombination aus einer Luxation des Gelenks mit einer dislozierten Radiusköpfchenfraktur und einem Abriss des Processus coronoideus bezeichnet und ist mit einer schlechten Prognose behaftet.

Insbesondere Resektionen des Radiusköpfchens führen aufgrund der medialen Bandinsuffizienz zu desolaten Ergebnissen. 


\section{Bandverletzungen im Wachstumsalter}

Im Kindesalter sind Bandverletzungen am Ellenbogen meist die Folge von Luxationsereignissen. Der Häufigkeitsgipfel liegt um das 10. Lebensjahr. Bandausrisse treten hierbei in bis zu $75 \%$ in Form von knöchernen Ausrissen auf. Bei unklaren konventionell-radiologischen Befunden kann die Kernspintomografie sinnvolle Informationen zur Therapieplanung bringen, um okkulte Frakturen und osteochondrale Läsionen darzustellen. Am häufigsten zeigt sich dabei eine Abrissfraktur des Epicondylus ulnaris.

\section{Therapie}

Nachweislich kommt es zu einer schnelleren Reintegrität der Weichteile bei frühfunktioneller Behandlung. Hierzu müssen bewegungsstabile Verhältnisse bei Luxationssicherheit und exakt reponiertem Gelenk bestehen.

Bereits am 3. Tag beginnt die Reparation im bestehenden Hämatom nach Bandruptur, nach 5-7 Tagen kommt es zur Bildung einer neuen, extrazellulären Matrix mit Gefäßeinsprossungen und der Einwanderung von Fibroblasten. Nach 2 Wochen ist das Granulationsgewebe bereits durch parallel verlaufende kollagene Fasern ersetzt. In der folgenden Phase des Remodellings kommt es über mehrere Monate hinweg zur Reifung der kollagenen Fasern. Zudem zeigt sich bei kurzen Ruhigstellungszeiten eine deutlich geringere Rate an Arthrofibrosen und Kontrakturen. Bei der Stabilitätsprüfung nach Luxation sollten stabile Verhältnisse bestehen. Bei instabilen Verhältnissen müssen die Begleitläsionen exakt evaluiert und das Ausmaß einer bestehenden Instabilität definiert werden.

\section{Konservative Therapie}

Die Reposition eines luxierten Ellenbogengelenks kann in der Regel mithilfe einer Analgosedierung problemlos durchgeführt werden.

Die Reposition sollte nach Möglichkeit unter Verwendung eines Bildverstärkers erfolgen, um das Ergebnis zu dokumentieren und die Stabilität zu prüfen. Essenziell ist die korrekte Einstellung der Ebene, insbesondere in der seitlichen Darstellung muss der Gelenkspalt frei einsehbar sein.

Für die Reposition erfolgt zunächst ein Zug am Unterarm in der HumerusLängsachse bei fixiertem Oberarm mit anschließender Bewegung des Unterarms in anteriorer Richtung mit leichter Bewegung in die Flexion. Bei der seltenen anterioren Luxation erfolgt die Reposition in gegenläufiger Richtung. Das Manöver sollte schonend durchgeführt werden. Bei frustranem Versuch sollte ein mögliches Repositionshindernis in Erwägung gezogen und keine weiteren Repositionsversuche unternommen werden.

Nach erfolgreichem Repositionsmanöver entscheidet die anschließende Stabilitätsprüfung unter dem BV über die weiter erforderlichen Therapieschritte. Der neurovaskuläre Status wird erneut erhoben und dokumentiert.

Bei stabilem Gelenk in vollem Bewegungsumfang und Varus- und Valgusstabilität genügt eine Ruhigstellung in einer Oberarmschiene in $90^{\circ}$ Beugung für ca. 1 Woche, forcierte Belastungen des Gelenks sollten für 6 Wochen vermieden werden.

Bei stabilen Verhältnissen zeigen die klinischen Ergebnisse nach Bandnaht keine besseren Resultate als bei konservativer Therapie.

Besteht im strecknahen Bereich bei weniger als $30^{\circ}$ eine Reluxationstendenz, sollte zunächst ebenfalls eine Ruhigstellung in $90^{\circ}$ Beugung erfolgen, nach $\mathrm{Ab}$ schwellung kann das Gelenk mit einer Bewegungsorthese geführt werden, wobei die Beugung freigegeben werden kann. Die Streckung bleibt zunächst limitiert und kann im Verlauf in Intervallen gesteigert werden.

Eine Ruhigstellung über den Zeitraum von 3 Wochen hinaus sollte aufgrund der Gefahr von persistierenden Bewegungseinschränkungen vermieden werden.

\section{Operative Therapie}

Operationsindikationen nach Ellenbogenluxation bestehen bei folgenden Befunden:

- Reluxationstendenz bei Beugung $>30^{\circ}$

- Repositionshindernisse

- dislozierte knöcherne Absprengungen und Frakturen (Kondylen, Epikondylen, Frakturen des Processus coronoideus Typ III, ggf. und Typ II)

- Reluxation nach zunächst konservativer Behandlung

- intraartikulär liegende Fragmente

- „Terrible Triad“

- offene Luxationen

- Gefäßnervenläsionen

- Kompartment
Ziel der operativen Versorgung ist es, die Kongruenz des Gelenks wiederherzustellen, das Gelenk zu stabilisieren und eine möglichst frühzeitige Bewegungstherapie einzuleiten, um bleibenden Funktions- und Bewegungseinschränkungen vorzubeugen.

\section{Versorgung von Kapselbandverletzungen}

Zunächst sollte die durch die Verletzung entstandene Einsicht in das Gelenk ausgenutzt werden, um das Hämatom auszuspülen und evtl. im Gelenkspalt befindliche Fragmente zu entfernen bzw. zu refixieren. Größere Gelenkfragmente (Knorpelabsprengungen) können gegebenenfalls mit resorbierbaren Pins oder unter Knorpelniveau versenkten Schrauben fixiert werden. Bandläsionen finden sich häufig im ansatznahen Bereich als Avulsionsverletzung bzw. als knöcherne Ausrisse. Diese lassen sich mit Fadenankern technisch einfach am ehemaligen Ansatzbereich refixieren. Alternativ besteht die Möglichkeit der transossären Refixation über entsprechende Bohrkanäle. Intraligamentäre Rupturen müssen bei Gewebsdefekten gegebenenfalls mit entsprechendem Nahtmaterial augmentiert werden. Rupturen des Lig. anulare radii können mitunter genäht werden, die Naht sollte jedoch nicht einengend sein und die Rotation des Radius limitieren. Bei ausgeprägten Instabilitäten kann die Refixation ausgerissener Kapselanteile, beispielsweise der ventralen Kapsel im Bereich des Processus coronoideus, zur Stabilisierung des Gelenks beitragen.

Ligamentär bedingte chronische Instabilitäten erfordern die Rekonstruktion der insuffizienten Bandanteile in Form von Ersatzplastiken durch autologes Sehnengewebe.

Diese erfordern ein hohes Maß an Erfahrung, da die isometrischen Verhältnisse und anatomischen Gegebenheiten exakt berücksichtigt werden müssen. Als Transplantat bietet sich idealerweise die Palmaris-longus-Sehne an. Alternativ kann die Plantarissehne, ein Streifen aus der Trizepssehne oder der mediale Rand der Achillessehne verwendet werden. Die Fixierung der Transplantate erfolgt mittels transossärem Durchzug. Radiale Instabilitäten, insbesondere des ulnaren lateralen Seitenbands (LUCL), können erfolgreich mit einer entsprechenden Ersatzplastik des Bandkomplexes therapiert werden. Posterolaterale Rotationsinstabilitäten Grad III und IV sollten 


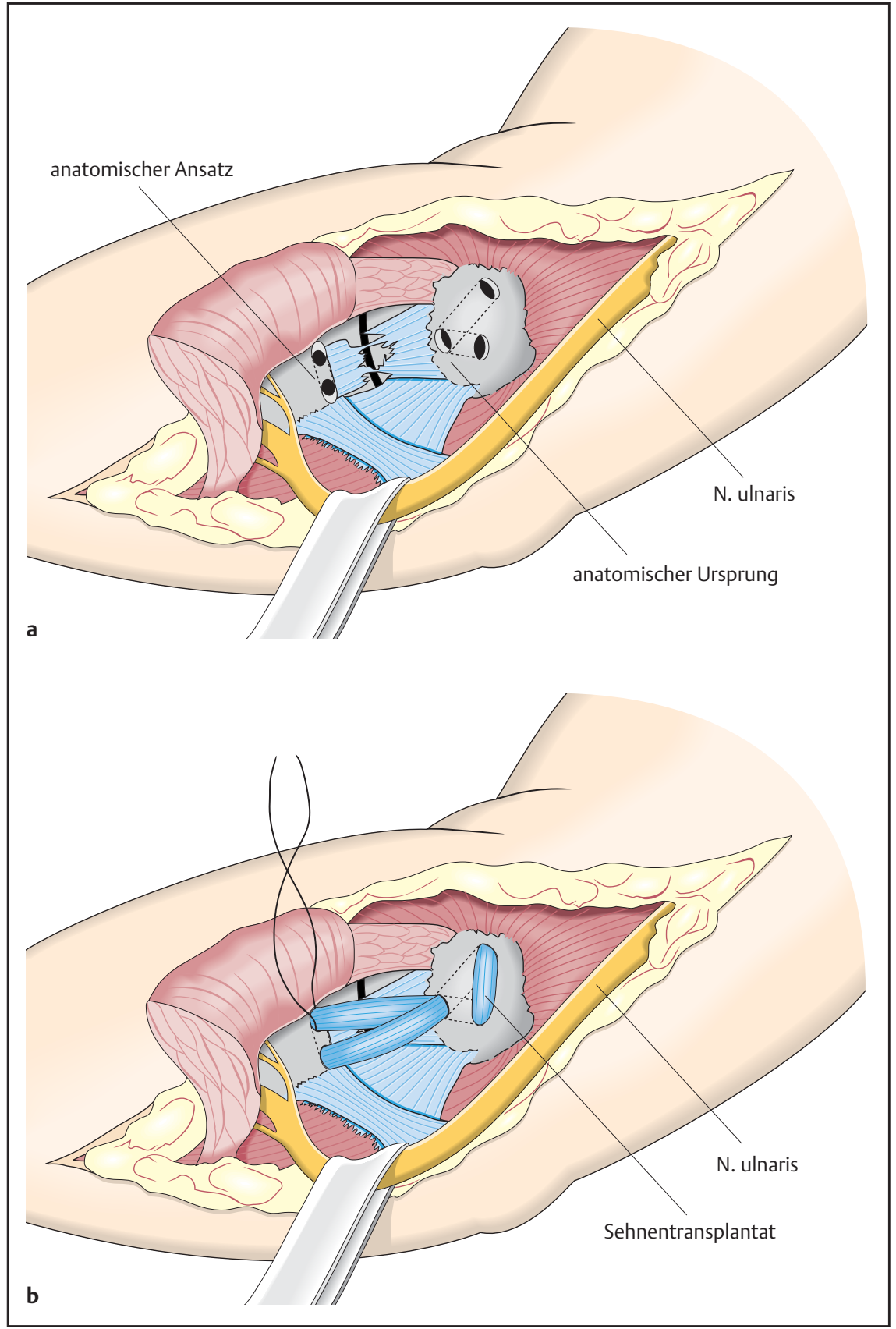

Abb. 4a und b a Ersatzplastik ulnarer Bandapparat mit freiem Sehnentransplantat, zunächst Anlage der transossär verlaufenden Bohrkanäle (aus: Miller, Howard, Plancher. Operationsatlas Sportorthopädie - Sporttraumatologie. 2003. S. 424. Mit freundlicher Genehmigung des Elsevier Verlags, Oxford). b Sehnentransplantat in situ (aus: Miller, Howard, Plancher. Operationsatlas Sportorthopädie - Sporttraumatologie. 2003. S. 425. Mit freundlicher Genehmigung des Elsevier Verlags, Oxford).

einer operativen Stabilisierung zugeführt werden. Für ein optimales Outcome kommt der Auswahl der Ansatzpunkte des Transplantats eine entscheidende Bedeutung zu, um der Isometrie der Bandführung gerecht zu werden. Die Technik nach Osborne/Cotterill kommt lediglich einer Raffung des meist schon ausgedünnten Gewebes gleich und führt zu weniger überzeugenden dann im Verlauf abhängig von der Stabilität freigegeben werden.

\section{Koronoidfrakturen}

Die Indikation zur Versorgung einer Koronoidfraktur ergibt sich aus der Kombination der begleitenden Verletzungen.

Insbesondere die Kombination mit Radiusköpfchenfrakturen kann schon die Refixation kleinerer Koronoidfragmente erforderlich machen. Bei Varus- und Valgusstabilität kommt der Stabilisierung der Kollateralbänder jedoch die entscheidende Rolle zu. Aufgrund der bereits oben erwähnten Bedeutung für die Gelenkstabilität müssen anteromediale Kantenfragmente am Koronoid evaluiert, reponiert und fixiert werden.

Typ-II-Verletzungen müssen bei Instabilität, insbesondere bei begleitender Radiusköpfchenfraktur und/oder begleitender medialer Kollateralbandläsion, versorgt werden. Typ-III-Läsionen gelten als instabil und müssen in der Regel osteosynthetisch versorgt werden.

Je nach Größe des Fragments bieten sich verschiedene Möglichkeiten zur Fixierung des Koronoids an. Die Osteosynthese kann mittels Zugschrauben direkt oder indirekt von dorsoulnar durchgeführt werden. Es sollte hierbei der $\mathrm{N}$. ulnaris dargestellt, angeschlungen und geschont werden. Für kleinere bzw. mehrfragmentäre Ausrisse, die mittels Schraubenosteosynthese nicht versorgt werden, besteht die Option zur Versorgung mit Nähten. Über retrograde Bohrkanäle kann eine Refixation des Koronoids in „Lassotechnik“ erfolgen, um den aufwendigeren ventralen Zugang zu vermeiden. Hierzu lassen sich kleine Ösendrähte als Fadendurchzugshilfe sinnvoll einsetzen (Abb. 5).

Bei größeren Substanzdefekten des Koronoids und dadurch bedingter Instabilität kann im Fall der Akutversorgung ein frakturiertes, nicht rekonstruierbares Radiusköpfchen verwendet werden, um den Processus coronoideus aufzubauen. Chronische Defekte können aus einem autologen Transplantat aus dem Beckenkamm rekonstruiert werden.

Zeigt sich im Rahmen der Rekonstruktion akuter Verletzungen nach wie vor eine instabile Situation, wird die Anlage eines Bewegungsfixateurs angeraten.

Dieser kann bei hoch instabilen Situationen zunächst in Beugung fixiert und 

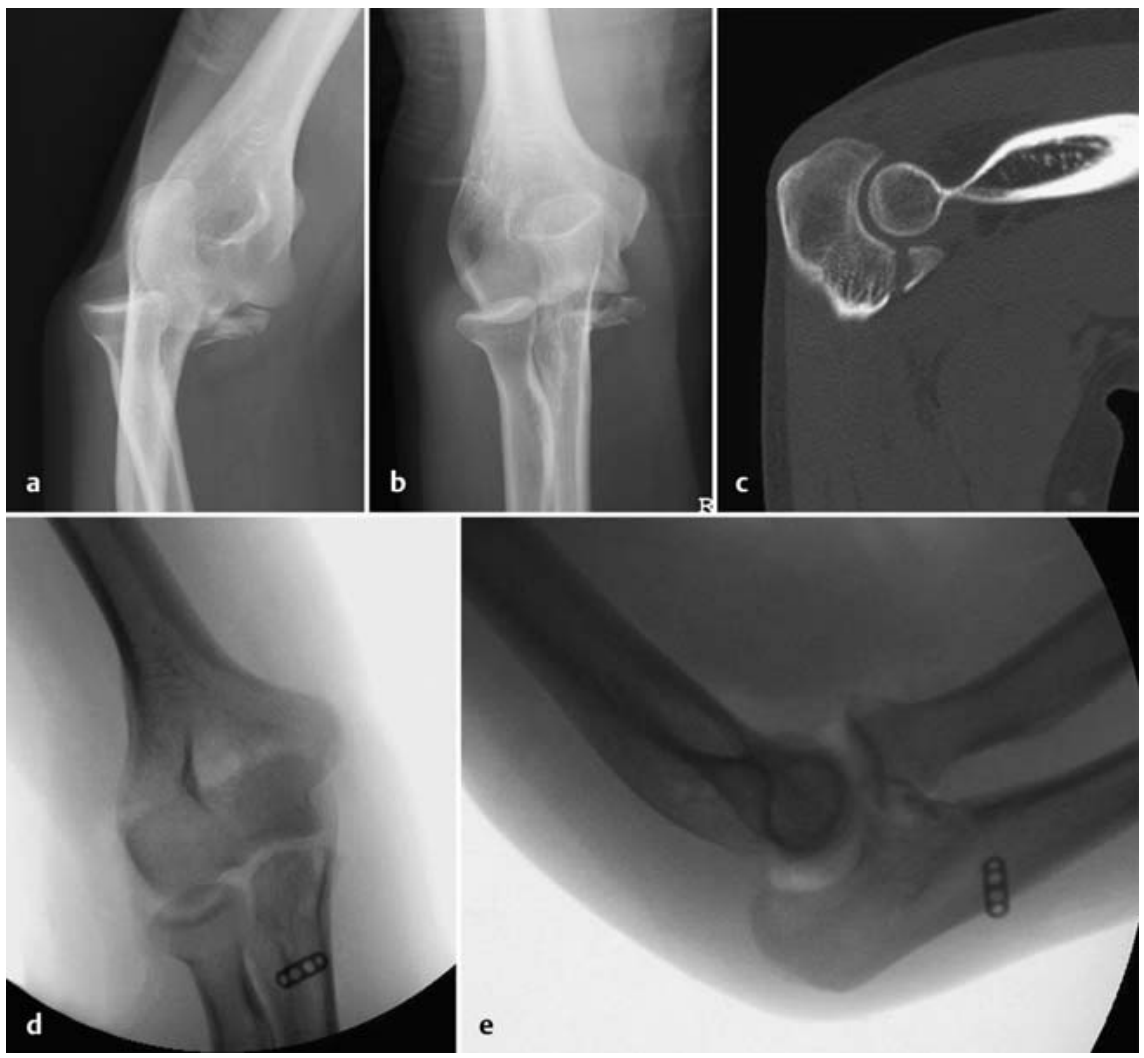

Abb. 5 a bis e a Fall 1: männl. 41 J., Snowboardsturz mit Ellenbogengelenksluxation, Röntgenbild nach Reposition a.-p. b Fall 1: seitl. Bild. c Fall 1: CT. d Fall 1: Röntgenkontrolle postop. a.-p. nach Refixation des Processus coronoideus in Lassotechnik und Naht des medialen Bandapparats. e Seitliches Bild postop.

\section{Radiusköpfchenfraktur}

Bei der Kombination einer Ellenbogenluxation mit einer Radiusköpfchenfraktur gilt es nach weiteren knöchernen und ligamentären Läsionen zu suchen. Neben der Versorgung der Begleitinstabilitäten ist ein stabil geführtes und kongruentes Humeroradialgelenk zu schaffen.

Ist die stabile osteosynthetische Rekonstruktion einer Radiusköpfchenfraktur nicht möglich, sollte bei Kollateralbandschäden keinesfalls nur eine Resektion durchgeführt werden. Dann besteht, insbesondere bei älteren Patienten, bei schlechter Knochenqualität oder bei instabiler Osteosynthese die Indikation zur Radiusköpfchenprothese.

Hierbei bieten neuere modulare Prothesenmodelle gute Möglichkeiten, die Implantatwahl an die anatomischen $\mathrm{Ge}$ gebenheiten anzupassen. Ziel ist es, das Radiusköpfchen exakt auf das Capitulum humeri zu zentrieren. Vermieden werden sollten zu große Durchmesser des Köpfchenimplantats und eine Verlängerung der radialen Säule. Bestehen im postoperativen Verlauf implantatbe- dingte Probleme, ist nach Heilung der ligamentären Strukturen und bei stabilen Bandverhältnissen eine Implantatentfernung vertretbar.

Die Resektion des Radiusköpfchens erfolgt am proximalen Rand der Tuberositas radii. Um eine Gefährdung des Ramus profundus des $\mathrm{N}$. radialis auszuschließen, erfolgt die Osteotomie mit der oszillierenden Säge unter dem Schutz von beidseits eingesetzten Hohmann-Haken.

\section{Terrible Triad}

„Terrible Triad“-Verletzungen gilt es zunächst sorgfältig zu diagnostizieren. So sollte bei jeder Ellenbogengelenksluxation mit Radiusköpfchenfraktur oder einer Fraktur des Processus coronoideus an die Möglichkeit einer Terrible-TriadVerletzung gedacht und der mediale Kollateralbandapparat überprüft werden. Um das optimale Ergebnis zu erreichen, ist eine stabile Situation im Humeroradialgelenk anzustreben, gegebenenfalls, auch wie oben beschrieben, durch einen Ersatz des Radiusköpfchens. Zudem soll- te eine Stabilisierung des Processus coronoideus und eine Rekonstruktion des medialen Kollateralbands erfolgen. In dieser Situation darf keinesfalls eine Resektion des Radiusköpfchens durchgeführt werden.

\section{Kindliche Bandverletzungen}

Wie oben erwähnt treten kindliche Bandverletzungen am Ellenbogen meist als knöcherne Bandausrisse, am häufigsten in Form von Frakturen des Epicondylus ulnaris auf. Da diese meist erheblich dislozieren, ist eine Reposition und Refixation erforderlich.

Hierzu bietet sich meist die Versorgung mit einer Zugschraube, ggf. die Fixierung mit einem Kirschner-Draht an. Die Metallentfernung kann nach 3-4 Monaten durchgeführt werden (Abb.6).

\section{Bewegungsfixateur}

Zur Anlage des Bewegungsfixateurs besteht bei Bandverletzungen des Ellenbogengelenks eine Reihe von Indikationen.

Ergibt die Rekonstruktion der ossären und ligamentären Verhältnisse nach Luxation keine zufriedenstellende Situation, kann durch die Anlage des Fixateurs nach passagerer Blockade des Bewegungsmechanismus zur Konsolidierung der Weichteile eine frühzeitige Bewegungstherapie ermöglicht werden.

Bei veralteten Instabilitäten oder rezidivierenden Luxationen kann mit dem Fixateur eine konzentrische Gelenkführung herbeigeführt werden. Nach Arthrolysen sowie bei Arthrofibrosen ist eine Distraktionsbehandlung des Gelenks möglich.

Ist eine akute Bandrekonstruktion bei komplizierter Luxation aufgrund einer Polytraumatisierung des Patienten oder anderer Ursachen nicht möglich, kann durch den Bewegungsfixateur eine kontrollierte Führung des Gelenks erreicht und eine Mobilisation des Gelenks ermöglicht werden.

Bei hoch instabilen Situationen wird durch die bilaterale Montage des Fixateurs eine höhere Stabilität erreicht.

Entscheidend für das Ergebnis der Versorgung mit dem Bewegungsfixateur ist neben einer optimalen Gelenkreposition und Rekonstruktion die korrekte Anlage der Rotationsachse. 


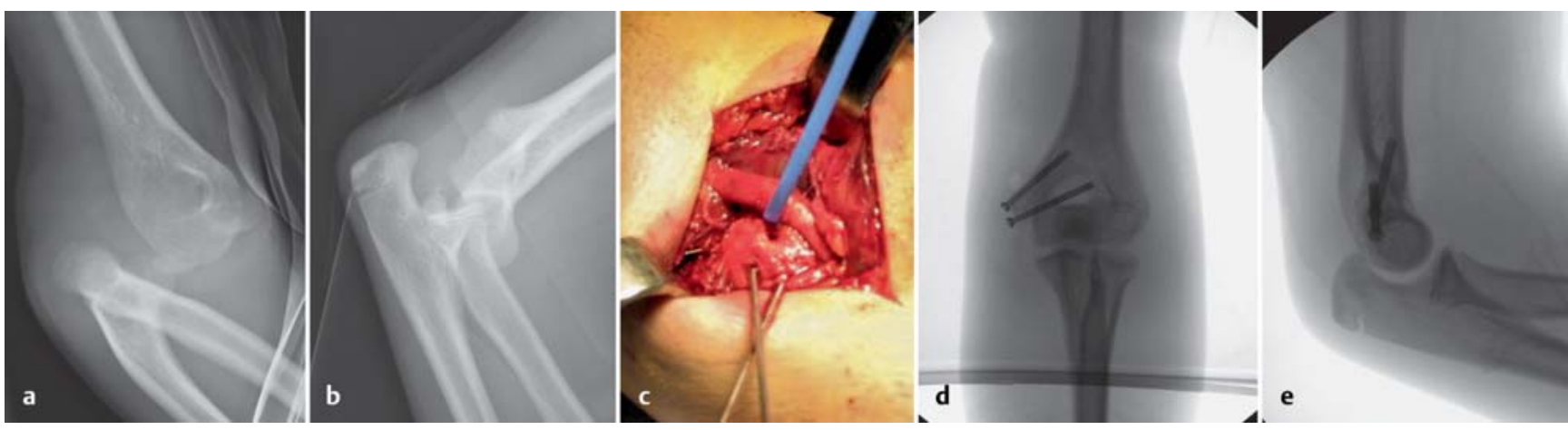

Abb. 6 a bis e a Fall 2: weibl. 11 J. Sturz von Schaukel, Ellenbogengelenksluxation mit Fraktur des Epicondylus ulnaris, Röntgenbild a.-p. b Fall 2: Röntgenbild seitlich. c Fall 2: Offene Reposition über ulnaren Zugang mit Darstellung des N. ulnaris (mit Loop angeschlungen), Fixierung mit Drähten zur Versorgung mit kanülierten Schrauben. d Fall 2: Röntgenbild postop. a.-p. e Fall 2: Röntgenbild postop. seitlich.

Hierzu muss eine exakt seitliche Projektion des Gelenks mit dem Bildverstärker eingestellt werden. Anschließend wird von lateral, zentral in der kreisrund zur Darstellung kommenden Trochlea und dem Capitulum humeri, die Spitze des Referenzpins aufgesetzt und unter Berücksichtigung der Ausrichtung in a.-p. Projektion der Pin eingebracht. Hierbei sollte der mediale Zielpunkt anterior und inferior des medialen Epikondylus liegen, um eine Läsion des Ulnarisnervs zu vermeiden.

Bereits geringe Abweichungen der Achse führen zu erheblichem Anstieg des Bewegungswiderstands.

Eine Abweichung des Rotationspunkts um $10 \mathrm{~mm}$ ergibt eine Erhöhung des Widerstands um Faktor 10. Anschließend wird der Fixateur auf den Pin geschoben, dabei sollte dieser unter Berücksichtigung der Weichteile möglichst knochennah platziert werden, um eine hohe Stabilität zu gewährleisten.

Unter Verwendung der Zieleinrichtung des Fixateurs wird zunächst die Pinanlage am distalen Humerus durchgeführt. Hierbei sollten die Weichteile weit genug eröffnet und schonend beiseite gehalten werden, um eine Schädigung des Radialisnervs zu vermeiden.

Nach Besetzen beider humeraler Pins erfolgt die Anlage der Pins an der proximalen Ulna, schließlich kann der Achspin entfernt werden. Damit wird durch die Rotation des Radius um die Ulna eine freie Pro- und Supination möglich. Eine temporäre statische trianguläre Fixierung kann mithilfe eines schräg verlaufenden Verbindungspins angelegt werden, welcher bei Bedarf zur Freigabe der
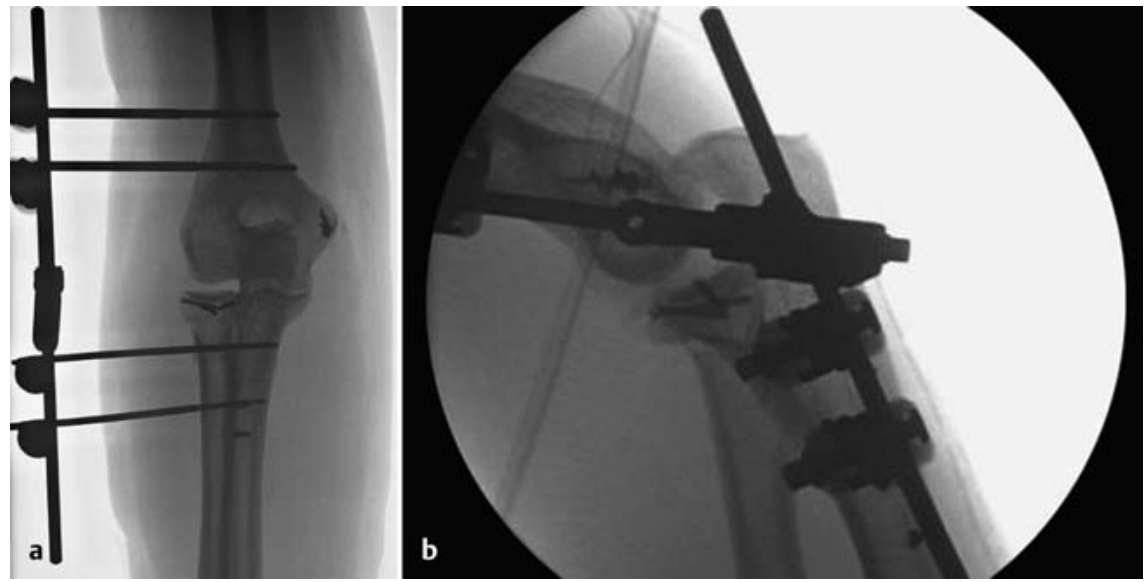

Abb.7a und b a Bewegungsfixateur nach Rekonstruktion bei komplexer Instabilität a.-p. b Bewegungsfixateur nach Rekonstruktion bei komplexer Instabilität seitl.

Beweglichkeit wieder entfernt werden kann (Abb. 7).

Nach Montage ist ein Durchbewegen des Fixateurs unbedingt durchzuführen und zu dokumentieren, um die exakte Führung und Einstellung des Gelenks zu kontrollieren.

\section{Lagerung}

Bewährt hat sich die Rückenlagerung mit Auslagerung der frei beweglichen oberen Extremität auf dem Armtisch. Insbesondere für den erweiterten posterioren Zugang kann in Rückenlage der Arm auf der Brust des Patienten, einem Kissen oder einer Tuchrolle aufliegend, gelagert werden. Empfohlen wird die Anlage einer Oberarmblutsperre. Für den rein posterioren Zugang bietet die Bauchlage eine gute Übersicht. Hierbei wird der Ellenbogen über einer weichen Rolle oder einem kleinen Seitentisch bei abduziertem Arm gelagert, sodass die Hand bei gebeugtem Ellenbogen herabhängt.

\section{Zugangswege}

Die Wahl des Zugangs hängt von der Lokalisation der Läsion ab. Eine präoperative BV-Untersuchung kann helfen, die erhobenen Befunde zu verifizieren und weitere Hinweise auf vorhandene Pathologien zu geben und schließlich bei der Auswahl des Zugangs behilflich zu sein.

\section{Lateraler (radialer) Zugang}

Für die Versorgung der zumeist mitbetroffenen radialen Bandstrukturen bietet sich der laterale Zugang an.

Nach längs verlaufender Schnittführung über dem radialen Gelenksbereich wird das Kocher-Intervall aufgesucht. Dieses liegt zwischen dem M. anconaeus und dem M. extensor carpi ulnaris und bietet eine gute Darstellbarkeit der radialen Bandstrukturen und des Radiusköpfchens. Über diesen Zugang kann, ventral am Radiusköpfchen vorbei, das Koronoid erreicht und mitversorgt werden. 


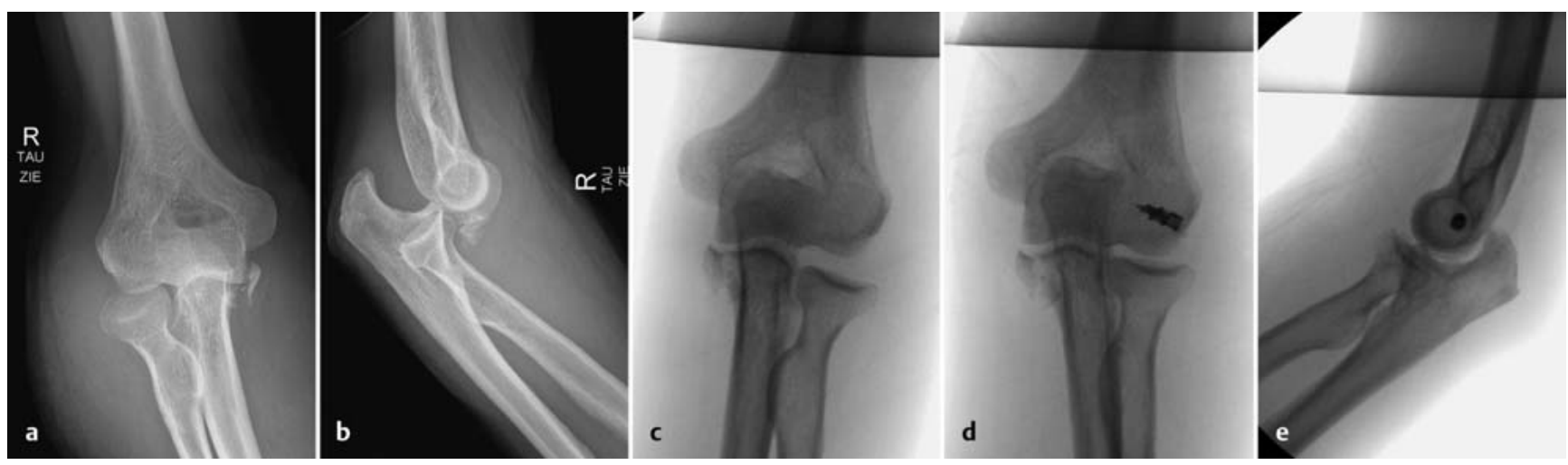

Abb. 8 a bis e a Fall 3: männl. 72 J. Luxation des Ellenbogengelenks bei Sturz auf glatter Straße, medial ulnar knöcherner Bandausriss und laterale intraligamentäre Bandruptur, Röntgenbild a.-p. b Fall 3: Röntgenbild seitl. c Fall 3. BV-Kontrolle nach Reposition lateral deutlich aufklappbar bei evidenter medialer Instabilität bei knöchernem Bandausriss. d Fall 3: Versorgungsbild postop. a.-p. mit Nahtanker radial und transossärer Refixation ulnar a.-p. e Fall 3: Versorgungsbild postop. seitl. mit Nahtanker radial und transossärer Refixation ulnar.

\section{Erweiterter posteriorer/ posteroradialer Zugang}

Dieser Zugang lässt sich in Rückenlage über den auf dem Bauch und mit einem Kissen oder Tuchrolle unterlegten Arm durchführen und kann als Erweiterung des radialen Zugangs erfolgen, um gegebenenfalls eine Versorgung im Bereich des Olekranons durchzuführen. Bei primär anzulegendem dorsalem Zugang ohne Indikation zur Erweiterung kann eine optimale Übersicht in Bauchlage erfolgen. Wird von diesem Zugang ein Eingriff nahe des Epicondylus ulnaris durchgeführt, muss eine Darstellung des Ulnarisnervs erfolgen. Wird ein Ablösen des M. supinator von der Ulna erforderlich, sollte das in Pronation des Unterarms erfolgen, um den tiefen Radialisast zu schonen.

\section{Medialer Zugang}

Über den ebenfalls längs verlaufenden medialen Zugang hat man eine gute Aufsicht auf die ulnaren Strukturen. Der $\mathrm{N}$. ulnaris muss hierbei sorgsam dargestellt und geschont werden, darf jedoch nicht vollständig entblößt und zu starkem Zug ausgesetzt werden.

Mit Gummizügeln, z.B. mit „Vesselloops“, kann er schonend zur Seite gehalten werden. Anschließend kann über eine Längsspaltung des M. flexor carpi ulnaris die Aufsicht auf die Kapselbandstrukturen erreicht werden. Anteromediale Bandausrisse sind über diesen $\mathrm{Zu}$ gang gut erreichbar, nach Mobilisation der Extensoren und Flexoren kann die Versorgung von Läsionen des Processus coronoideus durchgeführt werden (Abb. 8).

\section{Ventraler Zugang}

Der ventrale Zugang bietet eine eingeschränkte Aufsicht auf die ventral liegenden Strukturen und wird angewendet, wenn diese isoliert zu versorgen sind. Häufig sind die relevanten Strukturen bei radialen oder ulnaren Begleitpathologien über die dortigen Zugangswege problemlos mitzuversorgen. Es wird ein längs verlaufender, s-förmiger Hautschnitt angelegt, der proximal radial über dem Vorderrand des M. brachioradialis beginnt. Nach Ligatur von kreuzenden Venen findet sich nach Längsspaltung der Faszie zwischen M. brachioradialis und M. brachialis der sich in seinen motorischen und sensiblen Ast spaltende $\mathrm{N}$. radialis. Zusätzlich muss auf den $\mathrm{N}$. cutaneus antebrachii medialis, einen sensiblen Ast des N. musculocutaneus, geachtet werden. In der sich bietenden Loge zwischen Beugern und Streckern kann auf das Gelenk zugegangen werden. Zur Erweiterung des Zugangs kann die Sehne des M. brachialis eingekerbt werden, hierzu müssen jedoch die A. brachialis und der N. medianus dargestellt werden.

\section{Komplikationen/Aufklärung}

Neben den geplanten Zugangswegen erfolgt die Aufklärung über die allgemeinen OP-Risiken. Bei stabilisierenden Eingriffen muss die evtl. Erforderlichkeit der zusätzlichen Anlage eines Fixateur externe besprochen werden, um im Falle einer nicht ausreichenden Stabilisierung intraoperativ die Möglichkeit zur stabilen Gelenkführung offenzuhalten. Bei komplexen Verletzungen des Radiusköpfchens sollte die Option zum Ersatz des Radiusköpfchens offengehalten werden. Neben der allgemeinen Aufklärung über Nervenläsionen muss in Abhängigkeit vom Zugang eine Schädigung des $\mathrm{N}$. ulnaris, des N. radialis und auch des Ramus profundus des N. radialis, gegebenenfalls auch des N. medianus, thematisiert werden. Insbesondere beim selten verwendeten ventralen Zugang kann es, bei nicht sachgerechter Operationstechnik, zu Beeinträchtigungen der A. brachialis, mit dann evtl. erforderlichen Gefäßnähten oder entsprechenden Rekonstruktionen, kommen. Das Risiko für das Auftreten einer Arthrofibrose steigt erheblich bei Ruhigstellungen des Gelenks über einen Zeitraum von 2-3 Wochen hinaus.

Die Häufigkeit von heterotopen Ossifikationen wird in der Literatur im postoperativen/posttraumatischen Verlauf in 5$50 \%$ der Fälle angegeben.

Es wird ein multifaktoriell bedingtes Geschehen beschrieben, das besonders häufig nach komplexen Verletzungen mit erheblicher Weichteilschwellung und knöcherner Beteiligung auftritt. Komplexe Ellenbogenverletzungen in Kombination mit einem schweren Schädel-HirnTrauma weisen in bis zu 90\% der Fälle heterotope Ossifikationen auf. Durch Arthrolysen mit Abtragung der Ossifikationsherde kann eine Funktionsverbesserung erreicht werden. Speziell bei Verknöcherungen im Bereich der Membrana interossea kann der Bewegungsumfang für Umwendbewegungen erheblich erhöht werden. 


\section{Nachbehandlung}

Ziel nach jeder Versorgung ist, eine frühzeitige Bewegungstherapie des Gelenks zu ermöglichen, um die gefürchteten arthrofibrotischen Veränderungen mit konsekutiven Bewegungs- und Funktionseinschränkungen zu vermeiden.

Einfache Luxationen ohne Instabilität nach Reposition können frühfunktionell in vollem Bewegungsumfang therapiert werden. Bei Luxationsneigung in strecknaher Position kann das Gelenk mittels einer Bewegungsorthese unter Vermeidung des extensionsnahen Bereichs geführt werden. Eine Schiene wird lediglich für einige Tage bis zur Abschwellung angelegt.

Auch komplexe Versorgungen sollten keine Ruhigstellung über einen Zeitraum von 3 Wochen hinaus erfahren.

Zur postoperativen Schmerztherapie sollte bei Bedarf ein Plexuskatheter gelegt werden, um schmerzbedingte Bewegungseinschränkungen zu limitieren.

Zur stabilen Führung des Gelenks sollte die Behandlung mittels eines Bewegungsfixateurs über einen Zeitraum von 5-8 Wochen durchgeführt werden, gegebenenfalls kann das Gelenk nach Abbau des Fixateurs mit einer Orthese weiter geführt werden.

Aufgrund der schlechten funktionellen Ergebnisse bei Auftreten von heterotopen Ossifikationen kommt der Ossifikationsprophylaxe eine große Bedeutung zu.

Es wird eine 3-wöchige Indomentazintherapie mit 2-mal täglicher Gabe von $50 \mathrm{mg}$ empfohlen. Alternativ kann bei gegebener Infrastruktur eine postoperative Bestrahlung erfolgen. Diese sollte innerhalb der ersten 24 Stunden postoperativ durchgeführt werden, da hier die Differenzierung von pluripotenten Mesenchymzellen zu Osteoblasten stattfindet.

\section{Ergebnisse}

Die Qualität des funktionellen Ergebnisses korreliert stark mit der Schwere der ursprünglichen Verletzung. Einfache Luxationen bei jungen Patienten mit stabilen Verhältnissen nach Reposition zeigen optimale Ergebnisse. Mit der Schwere des Traumas und der Komplexität der Verletzung steigt die Rate an Einschränkungen der Funktionalität. Endgradige Bewegungseinschränkungen, insbesondere leichte Streckdefizite, werden in der Regel gut toleriert, da die meisten Bewegungen im Alltag in einem Bereich zwischen Extension/Flexion 0-30-130 und einer Pronation/Supination von 50-0$50^{\circ}$ ausgeführt werden.

Reluxationen und persistierende Instabilitäten treten nach fehleingeschätzten Verletzungen und nicht suffizient durchgeführter Primärtherapie auf.

Schlechte Ergebnisse sind besonders häufig bei „Terrible Triad“-Verletzungen zu sehen und verdeutlichen die Notwendigkeit einer optimalen Therapie.

\section{Schlussfolgerung}

Bandverletzungen des Ellenbogengelenks treten meist nach Luxationsereignissen auf. Häufig kommt es zu knöchernen Begleitverletzungen, deren Diagnostik und entsprechende Versorgung relevant für das klinische Outcome sind. Entscheidend für den weiteren Behandlungsweg ist die Beurteilung der Instabilität nach Reposition des Gelenks bzw. die Feststellung der Instabilitätsform bei chronischen Instabilitäten. Einfache Luxationen führen zu klinisch einwandfreien Ergebnissen. Mit der Schwere der Traumatisierung steigt die Rate an unbefriedigenden Resultaten. Aus diesem Grund ist eine exakte Diagnostizierung der Verletzung, Klassifizierung und Einleitung einer entsprechenden Therapie unerlässlich. Für die operative Versorgung komplexer Verletzungen ist ein hohes Maß an Erfahrung gefragt. Zur Vermeidung gefürchteter Bewegungseinschränkungen durch Arthrofibrosen sollte die Ermöglichung einer frühzeitigen Bewegungstherapie Ziel jeder Behandlung sein.
Ruhigstellungen des Gelenks über einen Zeitraum von 2-3 Wochen hinaus gilt es zu vermeiden.

\section{Literatur}

${ }^{1}$ Deml C, Arora R, Oberladstätter J et al. Funktionelle Therapie und ihre Grenzen bei akuten Ellenbogenluxationen. Unfallchirurg 2007: 110: 845-851

2 Frank J, Howorka A, Marzi I. Behandlung komplexer Ellenbogenverletzungen mit dem Bewegungsfixateur DJD II. Orthopäde 2011; 40: 316-322

${ }^{3}$ Frongia G, Günther P, Romero P et al. Ellenbogenluxationen im Kindesalter. Unfallchirurg 2012; 115: 125-133

${ }^{4}$ Hofmann D, Schüz W, Horas $U$. Traumatische Ruptur der A. brachialis durch eine geschlossene Ellenbogenluxation. Unfallchirurg 2011; 114: 816-821

${ }^{5}$ Kälicke T, Westhoff J, Wingenfeld C et al. Luxationsfrakturen des Ellenbogens mit Beteiligung des Processus coronoideus. Unfallchirurg 2003; 106: 300-305

${ }^{6}$ Kolb W, Guhlmann H, Windisch C et al. Komplexe osteoligamentäre Verletzungen des Ellenbogens. Unfallchirurg 2008; 111: 584-591

7 Lill H, Voigt C. Ellenbogenverletzungen. Chirurg 2004; 75: 1037-1051

8 Partenheimer A, Geisler A, Voigt C et al. Luxation, Instabilität und Luxationsfrakturen des Ellenbogengelenks. Trauma Berufskrankh 2007; 9 (Suppl. 2): S197-S201

9 Mittlmeier T, Beck M. Luxation des Ellenbogengelenks des Erwachsenen. Unfallchirurg 2009; 112: 487-505

10 Zimmermann G, Wagner C, Moghaddam A et al. Radiusköpfchenfraktur und Ellenbogenluxation. Trauma Berufskrankh 2004; 6: 297303

\section{Dr. med. Heinz Kusche \\ Oberarzt \\ Dr. med. Peter Gutsfeld}

Leitender Arzt

Abteilung für Unfallchirurgie und Sportorthopädie

Klinikum Garmisch-Partenkirchen in Kooperation mit der BG Unfallklinik

Murnau

Auenstraße 6

82467 Garmisch-Partenkirchen

heinz-kusche@web.de

Prof. Dr. med. Volker Bühren

Ärztlicher Direktor

BG Unfallklinik Murnau

Prof.-Küntscher-Straße 8

82418 Murnau 